\title{
Musculoskeletal Injuries and Disorders - A burden to Eswatini and Zimbabwe Sport
}

ISSN: 2576-8875

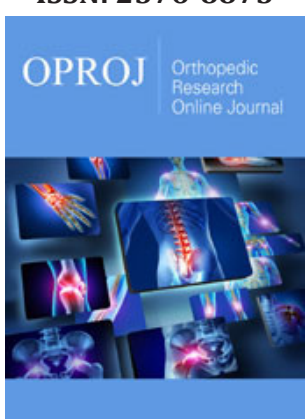

*Corresponding author: Adiele Dube, Department of Health Education, Southern Africa Nazarene University, Eswatini

Submission: 鲻 March 05, 2020

Published: 海 March 13, 2020

Volume 7 - Issue 1

How to cite this article: Dube $\mathrm{A}, \mathrm{Mdz}-$ iniso N.J. Musculoskeletal Injuries and Disorders - A burden to Eswatini and Zimbabwe Sport. Ortho Res Online J. 7(1) OPROJ.000652.2020.

DOI: 10.31031/OPROJ.2020.07.000652

Copyright@: Adiele Dube, This article is distributed under the terms of the Creative Commons Attribution 4.0 International License, which permits unrestricted use and redistribution provided that the original author and source are credited.

\author{
Adiele Dube ${ }^{1 *}$ and Mdziniso Ntombiyenkosi J ${ }^{2}$ \\ ${ }^{1}$ Department of Health Education, Southern Africa Nazarene University, Eswatini \\ ${ }^{2}$ Department of Physiotherapy, Mbabane Government Hospital, Eswatini
}

\section{Letter to Editor}

Musculoskeletal injuries, predominantly the hamstring, lower back and neck pain, are a challenge to amateur, professional and elite athletes across a wide range of sporting codes. Contact sports pose a high risk to the lower back and neck pain. Professionals such as sports medicine physicians, physiotherapists, athletic trainers and clinical researchers are concerned with these injuries. This is due to their high prevalence, high risk of recurrence, high pain and disability as well as high costs for treatment and rehabilitation. There is scant literature on injuries affecting Southern African sport population, however, researches carried among Eastern, Central and Western Europe reveal that in footballers the most prevalent muscle injury, with a high recurrence within a year after initial injury is the hamstring; rated between 12\%-33\% [1]. Disability-adjusted life years (DALYs) resulting from low back pain has also increased by 54\% [2] reaching as high as 84\% [3] between 1995 and 2017, where injured population are prone to experience more than one episode.

Platforms such as PubMed, Web of Science, Google Scholar, CrossRef and Lancet series, have published a number of researches on hamstring, lower back and neck pain. To date, no studies relating hamstring have specified how injury risk should be assessed when clinicians are faced with the return-to-play (RTP) decision after injury [1]. Similarly, the patho-anatomy of LBP is unclear often due to the uniqueness of most LBP-conditional abnormalities; therefore, the use of imaging appends very little hence be much less used [2]. The diversity in definitions and methodologies of RTP among sports medicine physicians and clinicians contributes significant differences in the results and conclusions obtained from sports injury research [4]. Basing on this, numerous stakeholders in sports injury and rehabilitation use varied methodologies based on their personal and institutional reasons to why RTP of hamstring and LBP should be accelerated or delayed [5]. With increasing literature, it is imperative to understand pain cognitions and central pain-modulating mechanisms so as to deal with LBP persistent disability and provide clarity on who is to be consulted and who is responsible for the RTP decision [1].

Globally, there are discrepancies and gaps between clinical practice and research, regarding the association of the extent of musculoskeletal injuries with time needed to recover, return to play or competition and specific measurements or parameters showing prognostic value. There is limited existence of use of consensually recommended first-line treatments, and inappropriately high use of imaging, rest, opioids, spinal injections and surgery [2]. Although not considered in few previous reports, the importance of neuromuscular coordination and the quality of interplay, between the different hamstring muscle bellies, among other factors, should be a key determinant within the intrinsic injury risk [6]. Although not yet studied and validated in clinical practice, the recent Delphi study may help sports medicine clinicians faced with the problem of when an athlete should RTP after a hamstring injury [1].

Musculoskeletal Injury and Disorders' (MIDs) unremittingly high incidence and recurrence rates indicate that the principal and underlying risk has not yet been fully identified. Despite that the causes of lower back pain are rarely addressed, at the moment low back pain is treated mainly with analgesics. Effective management of hamstring injury 
and acute back pain in the primary care setting can be the most important factors to help predict the time needed to RTP [6] and progression to chronic back pain [2]. Alternative treatments include physical therapy, rehabilitation and spinal manipulation; however, a broader recognition of multi-morbidity is necessary for assessment and treatment of MIDs, particularly in those with persistent problems. Literature have shown that rehabilitation through use of bio-psychosocial approach compared to biomedical (symptom focused) approach yield positive results in improving functionality when maintained under a healthier life-style $[2,7,8]$. Therefore, sports medicine physicians, physiotherapists, performance coaches, athletic trainers, clinical researchers and sports scientists should not only focus on pain symptoms and function, but also recognise accompanying problems such as painrelated fear, inactivity, anxiety, sleep disturbance, depression and related psychological stress.

It can be acknowledged that though clinical practice, research and policy-making often represent different worlds, they are always dependent upon each other. Multiple evidence-based guidelines for the management of hamstring grade 1 to grade 4 , acute and chronic back pains are readily available to guide providers, and therefore, adherence to such recommendations is important. Eswatini and Zimbabwe through their Ministries of Sports, Culture \& Youth/ Arts should improve their relationship and collaboration with the Ministries of Health (and Child Care) so that athletes' treatment becomes payable, accessible, effective and evidence-based. Treatment, RTP and prevention of recurrence through guidelines and standards are paramount, and policymakers should look at effectiveness, cost-effectiveness, necessity, feasibility, education and funding.

\section{Recommendations for future}

1. Stakeholders should join forces in building evidence basis,

2. Collaboration of Ministries; Sports, Culture \& Youth/Arts and Health (and Child Care)

3. Public health campaigns and seminars with messages about the importance of staying active, cognitive reassurance,

4. Athletic population is encouraged to stretch before and after exercising or physical activity

5. Treatment approach should be always updated through education systems, academic conference participation and research,
6. Physiotherapists and sports scientists are encouraged to use an effective spine manipulation treatment [9]

7. Apply the RTP model for hamstring injuries in football,

8. Use of Artificial and Emotional intelligence (AI and EI) for diagnosis and rehabilitation

RTP after musculoskeletal injuries decision should be considered as a multi-disciplinary decision. Athletic teams with limited access to comprehensive team staff are however advised to acknowledge the multifaceted nature of the RTP decision. Spinal manipulation should be considered as a one of the safe nondrug spine pain treatment. Professionals should put emphasis on pain relief, strength and flexibility assessment, mental and emotional readiness and functional performance of an athlete before returnto-play.

\section{References}

1. Horst N, Backx F, Goedhart EA (2017) Return to play after hamstring injuries in football (soccer): a worldwide Delphi procedure regarding definition, medical criteria and decision-making. British Journal of Sports Medicine 51(22): 1583-1591.

2. Kvåle Alice (2019) Musculoskeletal disorders - a challenge to society and to physiotherapists. European Journal of Physiotherapy 21(4): 185-186.

3. Russell LDO, Eric J Roseen DC, Rakel D (2018) Integrative Medicine, $\left(4^{\text {th }}\right.$ edn), Elsevier, Philadelphia, USA.

4. Junge A, Dvorak J (2000) Influence of definition and data collection on the incidence of injuries in football. Am J Sports Med 28(Suppl 5): 40-46.

5. Fuller CW, Ekstrand J, Junge A (2006) Consensus statement on injury definitions and data collection procedures in studies of football (soccer) injuries. Br J Sports Med 40(3): 193-201.

6. Crema MD, Godoy I, Abdalla RJ, de Aquino JS, Ingham S (2018) Hamstring injuries in professional soccer players: Extent of MRI-detected edema and the time to return to play. Sports health 10(1): 75-79.

7. Vibe Fersum K, O'Sullivan P, Skouen JS (2013) Efficacy of classificationbased cognitive functional therapy in patients with non-specific chronic low back pain: a randomized controlled trial. Eur J Pain 17(6): 916-928.

8. Vibe Fersum K, Smith A, Kvåle A (2019) Cognitive functional therapy in patients with non-specific chronic low back pain-a randomized controlled trial 3-year follow-up. Eur J Pain 23(8): 1416-1424.

9. Foster NE, Anema JR, Cherkin D (2018) Prevention and treatment of low back pain: evidence, challenges, and promising directions. Lancet 391(10137): 2368-2383.

For possible submissions Click below: 